\title{
Produção e bem-estar de búfalas (Bubalus bubalis) leiteiras: uma revisão
}

\section{Production and welfare of dairy buffaloes (Bubalus bubalis): a review}

\author{
Monique Valéria de Lima Carvalhal*, Franciely de Oliveira Costa \\ Doutoras em Zootecnia pela Universidade Estadual Paulista (Unesp), Jaboticabal, SP, Brasil
}

\section{Resumo}

A produção de búfalas leiteiras tem passado por uma crescente intensificação, o que tem promovido o aumento da produção de leite. Algumas das técnicas que fazem parte dessa intensificação podem expor os animais a alterações no ambiente físico, sendo potenciais causadoras de estresse. Tendo isso em vista, é importante ampliar o entendimento de como as rotinas de manejo podem influenciar o bem-estar e a produtividade das búfalas leiteiras, a fim de embasar recomendações de boas práticas de manejo que considerem o comportamento e o bem-estar desses animais. Aspectos que englobam a temática do bem-estar animal têm se destacado devido à crescente pressão dos mercados consumidores mais exigentes e à preocupação com as questões éticas relacionadas à criação de animais. Portanto, nessa revisão serão apresentadas informações sobre a produção e o bem-estar de búfalas leiteiras, com o objetivo de discutir a importância da avaliação de bem-estar animal nas fazendas comerciais.

Palavras-chave: Boas práticas de manejo. Qualidade do leite. Bubalinocultura.

\begin{abstract}
The production of dairy buffaloes has undergone an increasing intensification, which has promoted the increase of milk production. Some of the techniques that are part of this intensification can expose the animals to changes in the physical environment, being potential causes of stress. In view of this, it is important to broaden the understanding of how management routines can influence the welfare and productivity of dairy buffaloes in order to support recommendations of good handling practices that consider the behavior and welfare of these animals. Aspects encompassing the theme of animal welfare have gained space due to the growing pressure from the most demanding consumer markets and concerns with ethical issues related to animal husbandry. Therefore, in this review, the main aspects on dairy buffalo welfare will be addressed, seeking a reflection on the importance of the evaluation of animal welfare in commercial farms.
\end{abstract}

Keywords: Good handling practices. Milk quality. Buffalos production. 


\section{Introdução}

Os búfalos domésticos (Bubalus bubalis) contribuem significativamente para a produção mundial de leite (Borghese, 2005). É importante, portanto, entender como rotinas de manejo podem influenciar no bem-estar e na produtividade desses animais. Atualmente a produção de búfalos passa por um crescente incremento na mecanização dos sistemas de produção, com investimentos em inovações tecnológicas que visam a intensificação da produção leiteira. Algumas dessas técnicas podem expor os animais a alterações no ambiente físico, sendo potenciais causadoras de estresse (De Rosa et al., 2005) e de prejuízos ao bem-estar desses animais. Por conta deste risco, é fundamental expandir o conhecimento sobre as estratégias adaptativas dos búfalos antes de realizar mudanças nos sistemas de criação e no manejo, de forma a adequar recomendações técnicas às necessidades da espécie (Broom e Fraser, 2010).

É essencial que o manejo e o ambiente sejam adequados às necessidades e características das búfalas leiteiras a fim de não causarem estresse, o que poderia resultar em queda na produção e na qualidade do leite (Thomas et al., 2004) e, consequentemente, no seu bem-estar. Para Broom e Molento (2004), os efeitos sobre o bemestar incluem aqueles provenientes de doenças, traumatismos, fome, interações sociais, condições de alojamento, tratamento inadequado, manejo, transporte, procedimentos laboratoriais, mutilações variadas, tratamento veterinário ou alterações genéticas através de seleção genética convencional ou por engenharia genética. Assim, as práticas rotineiras realizadas na fazenda têm potencial para comprometer o bem-estar das búfalas. Além disso, há uma crescente pressão dos consumidores em relação ao bem-estar dos animais de produção, fazendo com que técnicos e produtores estejam mais atentos às questões éticas relacionadas ao modo de criação dos animais. Logo, nessa revisão serão discutidos aspectos do bem-estar de búfalas em lactação, tendo como objetivo propor uma reflexão sobre as necessidades desses animais nos sistemas de produção.

\section{A búfala e a produção de leite}

O bubalino (Bubalus bubalis) é uma espécie originária do continente asiático, que se difundiu para praticamente todos os continentes (Rodrigues et al., 2008) e que ocupa um relevante papel na produção de alimentos em diversos países, principalmente naqueles localizados em áreas tropicais (Bastianetto, 2009). Suas características zootécnicas (longevidade, adaptabilidade, prolificidade e precocidade) são consideradas uns dos principais motivos para a expansão do rebanho bubalino em muitos países do mundo (Borghese, 2005).

No Brasil, a Associação Brasileira de Criadores de Búfalos (ABCB) reconhece a existência de quatro raças, que diferem quanto à aptidão produtiva $\mathrm{e}$ distribuição pelas regiões do país: a) Jafarabadi - de dupla aptidão, com concentração na região sul do país e nos estados de São Paulo e Minas Gerais; b) Murrah - mais eficiente na produção de leite e derivados, concentrando-se também na região sul e estados de São Paulo e Minas Gerais; c) Mediterrâneo - caracterizado como animal de dupla aptidão, predominante na região nordeste do Brasil; d) Carabao - apesar de possuir dupla aptidão, é raramente usada para a produção de leite, sendo mais utilizada como força motriz; tem sua distribuição concentrada na Ilha do Marajó, no estado do Pará (Rodrigues et al., 2008).

A bubalinocultura está em significativa expansão em muitos países do mundo (Teixeira et al., 2005), sendo que a população de bubalinos mundial é estimada em 173 milhões de cabeças, com maior concentração na Índia, Paquistão e China. No Brasil, há cerca de 1,37 milhão de búfalos (IBGE, 2015), e em alguns estados a criação de búfalos está se tornando uma alternativa rentável de produção pecuária (Borghese, 2005). 0 estado de São Paulo, onde a população bubalina é de 250 mil animais, apresenta um crescimento anual do rebanho superior ao dos bovinos, na ordem de $2,8 \%$ ao ano (IBGE, 2011). Isso indica que a criação de búfalos tem sido considerada como nova opção produtiva, principalmente no que se refere à produção leiteira. Nesse estado, $40 \%$ dos rebanhos bubalinos estão voltados para esse tipo de produção (ABCB, 2004). 
Com relação aos aspectos quantitativos de produção de leite de búfala, observa-se maior potencialidade nas raças Murrah, Mediterrâneo e Jafarabadi (Nascimento e Carvalho, 1993). É possível encontrar rebanhos com média leiteira de 8 litros/dia, com produção anual de 1800 a $2000 \mathrm{~kg}$ em 270 a 300 dias de lactação (Valle, 1990). Sabe-se, ainda, que quando comparado ao leite bovino, o leite de búfala tem maiores teores dos principais constituintes, variando de $6,87 \%$ a 8,59\% em gordura e de 3,91\% a 4,55\% em proteína (Tonhati et al., 1998). Os valores para extrato seco total (gordura, açúcar, proteínas e sais minerais) encontrados na literatura variaram de 12,52\% a 18,99\% (Benevides et al., 2001). A lactose é o principal açúcar do leite e é a base para a obtenção dos derivados por meio da fermentação. Em bubalinos, os teores de lactose apresentam valores entre $4,8 \%$ e $5,5 \%$.

0 desenvolvimento desta cadeia produtiva pode trazer elevação de renda para os produtores, principalmente pelo fato de o leite de búfala conter elevado teor de sólidos totais, essencialmente a gordura, constituinte importante para a produção de derivados. Um dos fatores que atrai a atenção de novos investimentos neste setor é a valorização da mozzarella, além de medidas que vão desde campanhas de marketing até a certificação do produto, iniciadas pela Associação Brasileira de Criadores de Búfalos (ABCB) em 2009. Bernardes (2007) descreveu que a produção de derivados de leite de búfala gerou aos laticínios, no ano de 2006, uma receita da ordem de US\$ 55 milhões e, aos criadores, da ordem de US\$17 milhões. Além da mozzarella, há outros derivados que são produzidos a partir do leite de búfalas, tais como os queijos tipo minas frescal, ricota, doce de leite, queijo tipo coalho, iogurte e provolone.

É em regiões onde existe maior concentração de criadores (normalmente pequenos) que geralmente se implantam atividades de industrialização de derivados lácteos de búfalas. Nestas bacias leiteiras, gradativamente vem se observando a intensificação no manejo das búfalas, com adoção da prática de duas ordenhas diárias, suplementação com volumosos de melhor qualidade nos períodos de escassez de alimento e oferta de concentrados com base no nível produtivo dos rebanhos (Bernardes,
2007). Concomitantemente, observa-se em alguns rebanhos intensificação do sistema de criação, com utilização de ordenha mecânica, manejo intensivo de pastagem (com adoção de tecnologias como adubação, irrigação e pastejo sob lotação rotativa), oferecimento de dieta concentrada no cocho, entre outros. A combinação dessas ações tem permitido aos criadores alcançarem níveis produtivos significativamente mais elevados. Não há dúvidas de que essas mudanças nos sistemas de produção têm proporcionado ganhos econômicos e sociais importantes, porém ainda há questionamentos quanto ao seu impacto no grau de bem-estar dos animais.

\section{Bem-estar de búfalas leiteiras}

Estudos de etologia aplicada focados na cognição, motivação e complexidade do comportamento social nos animais domésticos levaram a um rápido desenvolvimento da ciência do bem-estar animal (Broom, 2011). Após o conceito de bem-estar animal ser implantado no cenário da produção animal, foram definidos protocolos de boas práticas de manejo que ofereceram recomendações técnicas de como realizar os manejos promovendo o bemestar dos animais.

Assim, o bem-estar dos animais de fazenda passou a fazer parte dos critérios de qualidade e segurança alimentar dos produtos de origem animal. Dentre os fatores considerados pelos consumidores estão: (a) o bem-estar dos animais utilizados na produção; (b) os impactos sobre o meio ambiente, incluindo a conservação da flora e da fauna nativas; (c) a remuneração justa aos produtores, especialmente nos países emergentes; (d) a preservação das comunidades rurais (Broom, 2010). Essas mudanças com relação às exigências do mercado consumidor impuseram novos desafios à produção animal e, consequentemente, impulsionaram a busca por métodos de criação que garantam qualidade de vida aos animais. Em muitos aspectos, a melhoria no grau de bem-estar dos animais leva também a melhorias na produtividade (Broom e Fraser, 2010).

Bem-estar animal é definido pelo estado do indivíduo em suas tentativas de se ajustar 
ao ambiente (Broom, 1986). Do ponto de vista científico, trata-se de um estado mensurável, e qualquer avaliação deve ser feita de forma objetiva, independente de considerações éticas (Broom e Molento, 2004). O grau de bem-estar dos animais depende do tipo e intensidade dos desafios enfrentados por eles, e para identificar esses desafios é importante uma visão integrada do sistema produtivo (Broom e Fraser, 2010). Por esse motivo, é essencial adotar uma abordagem que leve em conta todos os elementos que tenham potencial para prejudicar a qualidade de vida dos animais. A avaliação do bem-estar animal é realizada utilizando-se indicadores baseados no ambiente e nos próprios animais, que ajudam a identificar o estado de bem-estar de um dado animal, podendose adotar uma escala que varia de muito bom a muito ruim (Broom e Fraser, 2010).

Existem diferentes metodologias utilizadas para a avaliação do bem-estar animal, sendo uma delas o protocolo desenvolvido pelo projeto Welfare Quality ${ }^{\circledR}$ (2009), o qual teve como objetivo fornecer parâmetros para uma avaliação objetiva do bemestar animal. Com base no esquema de avaliação do Projeto Welfare Quality ${ }^{\circledR}$ utilizado para vacas leiteiras, De Rosa et al. (2007a) adaptaram os indicadores de bem-estar para sua aplicação às búfalas leiteiras, como descrito no Quadro 1.

Quadro 1 - Princípios, critérios e medidas de avaliação para o bem-estar de búfalas leiteiras (Welfare Quality ${ }^{\circledR}$, adaptado por De Rosa et al., 2007a)

\begin{tabular}{|c|c|c|}
\hline Princípio & Critério & Medida \\
\hline \multirow{2}{*}{ Boa alimentação } & 1. Ausência de fome prolongada & Porcentagem de animais muito magros, normais e muito gordos. \\
\hline & 2. Ausência de sede prolongada & $\begin{array}{l}\text { Número de bebedouros; } \\
\text { Higiene dos bebedouros. }\end{array}$ \\
\hline \multirow{3}{*}{ Bom alojamento } & 3. Conforto em relação ao descanso & $\begin{array}{l}\text { Tempo necessário para os animais se deitarem; } \\
\text { Porcentagem de animais que se encostam nas instalações ao se deitarem; } \\
\text { Porcentagem de animais que se deitam com o quarto traseiro para fora da } \\
\text { cama. }\end{array}$ \\
\hline & 4. Conforto térmico & $\begin{array}{l}\text { Presença de sistemas para termorregulação (poças d'água, lagos naturais, } \\
\text { piscinas artificiais, chuveiros). }\end{array}$ \\
\hline & 5. Facilidade de movimento & Liberdade de movimentação. \\
\hline \multirow{3}{*}{ Boa saúde } & 6. Ausência de lesões & $\begin{array}{l}\text { Porcentagem de animais com problemas de locomoção (claudicação); } \\
\text { Porcentagem de animais com alterações no tegumento, principalmente na } \\
\text { região do úbere. }\end{array}$ \\
\hline & 7.Ausência de doenças & $\begin{array}{l}\text { Porcentagem de animais com sinais clínicos de doenças como tosse, } \\
\text { corrimento nasal, ocular ou vaginal, diarreia, frequência respiratória } \\
\text { aumentada e prolapso uterino. }\end{array}$ \\
\hline & 8. Ausência de dor induzida por manejo & $\begin{array}{l}\text { Uso ou não de anestésicos e/ou analgésicos em procedimentos de manejo } \\
\text { aversivos (descorna, castração, corte de cauda e ponta da língua). }\end{array}$ \\
\hline \multirow{4}{*}{$\begin{array}{l}\text { Comportamento } \\
\text { apropriado }\end{array}$} & 9. Expressão de comportamento social & $\begin{array}{l}\text { Frequência de comportamentos sociais positivos (lamber e coçar); } \\
\text { Frequência de comportamentos sociais negativos (cabeçadas, deslocamentos e } \\
\text { disputas severas). }\end{array}$ \\
\hline & 10. Expressão de outros comportamentos & $\begin{array}{l}\text { Frequência de animais com estereotipias (sucção e mordedura das instalações } \\
\text { e/ou dos outros animais). }\end{array}$ \\
\hline & 11. Boa interação humano-animal & $\begin{array}{l}\text { Avaliação Qualitativa do Comportamento (do inglês Qualitative Behaviour } \\
\text { Assessment, QBA). }\end{array}$ \\
\hline & 12. Ausência de medo & Teste de distância de fuga no momento da alimentação. \\
\hline
\end{tabular}


Cada medida dentro do critério recebe uma pontuação ponderada de acordo com seu grau de importância. Segundo os organizadores do projeto, o grau de importância de cada medida deve ser definido previamente à aplicação do protocolo por uma equipe de pesquisadores (Welfare Quality ${ }^{\circledR}$, 2009). Para as búfalas, por exemplo, ainda são necessários mais estudos com relação à ponderação das medidas. Em geral, o protocolo auxilia na identificação dos principais pontos a serem medidos, sugerindo que cada produtor defina o intervalo mínimo e máximo de cada medida, uma vez que esse aspecto ainda não foi definido para essa espécie. Os resultados obtidos para cada uma das diferentes medidas são combinados para calcular a pontuação final obtida em cada critério e, consequentemente, em cada princípio. No final é obtida uma pontuação em uma escala entre zero e 100 , sendo que zero representa mais problemas de bem-estar e 100 a melhor situação possível.

Atualmente, há uma proposta em desenvolvimento para avaliação de risco no bem-estar de búfalas leiteiras criadas em confinamento no Instituto Zooprofilatico Sperimentale del Mezzogiorno (IZSM), localizado na Itália. 0 projeto é intitulado "Ruminant Welfare ${ }^{\circledR ”}$ e envolve a avaliação das questões de bem-estar relacionadas aos animais, além de englobar avaliações de biossegurança na propriedade. Recentemente este protocolo começou a ser utilizado na região italiana da Campânia e está em fase de adaptação (Vecchio et al., 2018). No nosso conhecimento, este é o único protocolo de avaliação de bem-estar desenvolvido especificamente para os búfalos. No entanto, até a presente data, não há publicações científicas com a apresentação do método de avaliação.

Existe outra abordagem para o direcionamento da avaliação objetiva do bem-estar animal que foi descrita por Mellor e Reid (1994). Para esses autores, a avaliação do bem-estar animal deve englobar quatro domínios principais relacionados à nutrição, ambiente, saúde e comportamento. Incluem, ainda, um quinto domínio, referente aos estados mentais dos animais, influenciado por todos os estímulos internos e externos que afetam o animal nos quatro primeiros domínios (Mellor, 2017). Este último aspecto é o que caracteriza a principal diferença entre esse modelo de avaliação e o protocolo Welfare Quality ${ }^{\circledR}$. Considerando que todo o processo de criação das búfalas leiteiras é constituído por rotinas de manejo que envolvem os quatro domínios (nutrição, ambiente, saúde e comportamento) e que, juntos, eles podem afetar o estado mental dos animais, é indispensável a adoção de um sistema de monitoramento do bemestar. A partir dos resultados obtidos em avaliações rotineiras de bem-estar será possível aperfeiçoar as técnicas de manejo utilizadas, garantindo melhores respostas nesses quatro domínios e, potencialmente, estados mentais positivos aos animais.

Segundo Napolitano et al. (2013), alguns entraves para a aplicação da avaliação do bem-estar nas búfalas leiteiras se devem à falta de conhecimento tanto em temas básicos, aspectos fisiológicos e anatômicos desses animais, como em alguns temas mais aplicados do comportamento dos búfalos. Esses autores também enfatizaram a necessidade de novos estudos que descrevam indicadores de bem-estar próprios para esta espécie, uma vez que o protocolo de avaliação focado na criação de búfalas leiteiras foi adaptado dos protocolos criados para bovinos leiteiros e, até, para bovinos de corte.

Dentre as principais adaptações realizadas no protocolo Welfare Quality ${ }^{\circledR}$ para essa espécie, podese citar a avaliação do primeiro critério, no qual se sugere a utilização do escore corporal adaptado do protocolo de bovinos de corte ou de raças de dupla aptidão. Além disso, para a avaliação da limpeza corporal, os autores destacam alguns cuidados necessários referentes à caracterização da sujeira presente no corpo do animal (Napolitano et al., 2013). Em muitas fazendas os búfalos têm acesso à água para imersão e isso faz com que, normalmente, seu corpo esteja coberto por lama, o que contribui para a termorregulação desses animais. Por outro lado, a presença de lama pode indicar uma baixa frequência de higiene das instalações e também pode estar associada à alta densidade, o que é prejudicial aos animais.

Outro ponto importante a ser destacado diz respeito às alterações de tegumento principalmente na região do úbere, já que nas búfalas é comum a aplicação de ocitocina exógena durante a ordenha, o que pode provocar um abscesso e prejudicar de diversas formas o bem-estar desses animais. 
Outra diferença destacada no protocolo criado especificamente para os búfalos está relacionada à utilização do anel de desmame nos bezerros. $\mathrm{Na}$ Itália, além da utilização do anel, um problema comum é o corte da ponta da língua dos bezerros, prática considerada aversiva, no intuito de evitar a amamentação cruzada (De Rosa et al., 2007b).

No Brasil, especificamente, observa-se uma dificuldade da aplicação do método em sistemas de criação extensivos de bubalinos leiteiros. Nesses casos há a necessidade de adaptar algumas das medidas, já que o protocolo contempla indicadores exclusivos para avaliação de sistemas intensivos de produção, que confinam os animais em instalações fechadas (Garcia, 2013).

Relatando as maiores dificuldades encontradas após a aplicação do Welfare Quality ${ }^{\circledR}$ em fazendas italianas, De Rosa et al. (2007b) descreveram que os principais problemas estão associados ao princípio de "instalações inadequadas". A maior parte das fazendas italianas mantém as búfalas confinadas e, geralmente, com espaço para movimentação bastante reduzido, o que acaba interferindo no tempo de descanso e no bem-estar dos animais. A restrição de espaço apresenta consequências físicas, como a locomoção limitada, danos aos membros, espaço para descanso reduzido, e psicológicas, como interações agonísticas forçadas e distância de fuga reduzida (Maton e Daelemans, 1989). Diversos estudos têm avaliado o impacto da restrição de espaço no comportamento, no bem-estar e na produção de búfalos (Grasso et al., 2003; Grasso et al., 2004; Napolitano et al., 2004; Tripaldi et al., 2004; De Rosa et al., 2007b). De Rosa et al. (2007b) avaliaram o comportamento de búfalas submetidas a dois tipos de sistemas de criação. Dezesseis animais foram submetidos a alojamento em baia de $3 \mathrm{~m}^{2}$, com uma área em piquete de mais $3 \mathrm{~m}^{2}$ (IR). Outros 16 animais foram mantidos em uma área de, aproximadamente, 40 hectares (ER). Ao realizar teste de novo objeto, esses autores observaram que os animais criados na área menor (IR) exploraram mais o objeto utilizado devido à falta de estímulo no ambiente onde viviam. Enquanto isso, o outro grupo de búfalas (ER) apresentou menor interesse pelo estímulo (novo objeto), uma vez que estavam submetidas a um ambiente muito mais complexo. Os autores também aplicaram um teste de distância de fuga ao manejador e observaram que as búfalas alojadas na grande área (ER) apresentaram menor distância de fuga do que aquelas confinadas em espaço restrito (IR). Diante desses resultados, os autores concluíram que as búfalas do grupo ER foram menos reativas ao novo estímulo e desenvolveram uma melhor relação humanoanimal. Ou seja, o espaço no qual os búfalos são criados pode interferir na capacidade desses animais em enfrentar desafios.

A disponibilidade de espaço também pode interferir nos níveis de cortisol, o que, por sua vez, pode estar relacionado com a produção desses animais, como observado por Tripaldi et al. (2004), que avaliaram o comportamento, as respostas imunológicas, a concentração de cortisol e de metabólitos, e a produção de leite de búfalas leiteiras submetidas a dois tipos de tratamento (baia de $10 \mathrm{~m}^{2}$ por animal e baia de $10 \mathrm{~m}^{2}$ com área aberta de $500 \mathrm{~m}^{2}$ por animal). Estes autores observaram que os animais mantidos na área de espaço reduzido apresentaram maior período de inatividade. Além disso, os níveis de cortisol foram mais altos para as búfalas submetidas a este mesmo tratamento (com restrição de espaço). Por outro lado, as respostas imunológicas, a concentração dos metabólitos e a produção de leite não foram afetadas pelo tratamento. A partir desses resultados, os autores concluíram que o fornecimento de um sistema de alojamento que proporcione condições mais próximas às naturais foi capaz de melhorar o bem-estar das búfalas, por possibilitar a estas a expressão de alguns comportamentos espécie-específicos naturais.

Em relação ao conforto térmico, os búfalos têm necessidade de se proteger da radiação solar direta, utilizando, por exemplo, poças de água ou lama para se refrescarem nos períodos quentes do dia (Garcia et al., 2011). Em estudo realizado por Ablas et al. (2007), foram avaliadas as respostas comportamentais de bubalinos em relação à capacidade de termorregulação com e sem acesso à sombra natural e à água para imersão em criação a pasto. Todas as búfalas utilizaram algum tipo de proteção (sombra ou água para imersão) contra o calor nas horas mais quentes do dia, e quando esses dois tipos de proteção estiveram disponíveis, as búfalas preferiram a água para imersão. Esse resultado mostra que a disposição de lagoas ou açudes representa um 
recurso benéfico aos animais. Nas condições climáticas do Brasil, é importante ter em mente a necessidade de se disponibilizar sombra natural ou artificial a esses animais. Nesse sentido, Garcia et al. (2011) descreveram que o sombreamento em um sistema silvipastoril diminuiu significativamente a frequência cardíaca e a temperatura retal de búfalas leiteiras. Para os autores, em 71,4\% das observações, os animais mantidos em sistema silvipastoril com sombreamento apresentaram índices de conforto próximos ao ideal.

Considerando 0 aspecto comportamental, búfalas leiteiras têm necessidade de expressar seus comportamentos naturais, como poder percorrer longas distâncias durante o dia, necessidade que está diretamente ligada à disponibilidade de espaço. Carvalhal et al. (2017) observaram que búfalas leiteiras percorreram diariamente, em média, 7413 $\pm 1039 \mathrm{~m} /$ dia (média \pm desvio padrão), variando de 5762 a $10124 \mathrm{~m} /$ dia, mostrando a relevância do comportamento exploratório para essa espécie. 0 resultado encontrado por esses autores foi superior à distância média percorrida relatada por Sabia et al. (2014) $(2,1 \pm 0,3 \mathrm{~km}$ por dia). Além desses achados, Carvalhal et al. (2017) não encontraram relações significativas entre a distância média percorrida por dia e a produção e a qualidade (gordura e proteína) do leite de búfalas, indicando que essa atividade exploratória não afeta os índices produtivos desses animais.

Outro aspecto que deve ser levado em conta é a dificuldade de adaptação das búfalas leiteiras para a ordenha sem bezerro ao pé, o que resulta em falhas na descida do leite, com implicações negativas no bem-estar das búfalas e na eficiência da ordenha. Sabe-se que as búfalas são sensíveis a pequenas mudanças na rotina de ordenha, influenciando a liberação de ocitocina e a produção de leite (Thomas et al., 2005). Essas mudanças podem envolver aspectos relacionados ao contato mais próximo aos manejadores, ruídos dos equipamentos e do manejo, separação da cria, mudança no grupo social, entre outros. No intuito de reduzir o estresse dos animais no primeiro contato com essas mudanças, Polikarpus et al. (2014) avaliaram o efeito da habituação pré-parto de 16 novilhas bubalinas sobre o comportamento e o desempenho desses animais. Neste estudo, o processo de habituação consistiu em conduzir os animais à sala de ordenha, onde permaneceram por 10 minutos sem serem tocados. Após esse período, iniciava-se a lavagem do úbere com água morna e secagem com papel descartável. Os autores observaram menor expressão de passos e coices ao longo da lactação por parte dos animais que receberam a habituação pré-parto. Com relação à produtividade, não foram encontradas diferenças significativas na qualidade do leite e no fluxo lácteo entre os grupos habituados e não habituados. Sendo assim, a habituação pré-parto das novilhas bubalinas pode auxiliar na redução do estresse desses animais durante a primeira lactação e, potencialmente, auxiliar na redução da aplicação de ocitocina exógena.

Em estudo realizado por Cavallina et al. (2008) foi demonstrado o impacto do uso da ordenhadeira mecânica no bem-estar de búfalas leiteiras, especialmente primíparas, considerando como indicadores de estresse agudo as alterações nas frequências de chute, retirada do conjunto de teteiras no momento da ordenha, defecação, micção e administração de ocitocina. A necessidade de administração de ocitocina em búfalas leiteiras foi considerada um possível indicador de bemestar relacionado a uma interação humano-animal inadequada (De Rosa et al., 2005). Saltalamacchia et al. (2007) observaram que o comportamento do manejador esteve relacionado ao comportamento das búfalas na ordenha, que, por sua vez, esteve relacionado à aplicação de ocitocina.

A aplicação de ocitocina exógena é uma prática que vem se tornando rotineira nos rebanhos de búfalas leiteiras, nos quais não se realiza a ordenha com bezerro ao pé. Sabe-se que esses animais, quando comparados a bovinos de raças especializadas, têm maior dificuldade para ejeção do leite (Thomas et al., 2005; Mello et al., 2016) e, por isso, utiliza-se a ocitocina para facilitar este processo. Destacamos a importância de se avaliar os riscos inerentes à aplicação de medicamentos intravenosos como, por exemplo, os riscos de transmissão de doenças infectocontagiosas entre as búfalas do rebanho com a utilização de uma única agulha compartilhada, além de ocorrências de processos inflamatórios nas veias mamárias (Mello et al., 2016). Assim, recomendamos a adoção conjunta de boas práticas de manejo, tanto na 
preparação de novilhas para a primeira ordenha quanto na rotina da ordenha, de forma a facilitar a adaptação dos animais aos manejos da fazenda, além de selecionar animais com maior facilidade de ejeção do leite sem bezerro ao pé.

\section{Conclusão}

No Brasil, o búfalo é reconhecido pela maioria das pessoas envolvidas na cadeia produtiva de leite como um animal "rústico", que tem a capacidade de se adaptar e manter sua produção em qualquer condição ambiental, até mesmo em escassez de alimento. Por conta disso, na maioria das vezes, devido à falta de conhecimento com relação às necessidades desses animais pelos produtores, há descaso em relação aos cuidados com os búfalos, o que acaba refletindo em baixos índices zootécnicos e baixo grau de bem-estar animal. Nesse sentido, estudos sobre o comportamento e o bem-estar de búfalos se tornam extremamente necessários, uma vez que estes são uma importante ferramenta para o desenvolvimento dos sistemas de criação e servem de base para a identificação de medidas eficazes para a avaliação do bem-estar desses animais.

\section{Referências}

Associação Brasileira de Criadores de Búfalos (ABCB). Desempenho da produção dos laticínios com selo de pureza $100 \%$ búfalo. 2004 [acesso 26 out 2017]. Disponível em: https://tinyurl.com/yb25monc.

Ablas DS, Titto EAL, Pereira AMF, Titto CG, Leme TMC. Comportamento de bubalinos a pasto frente a disponibilidade de sombra e água para imersão. Cienc Anim Bras. 2007;8(2):167-75.

Bastianetto E. Criação de búfalos no Brasil: situação e perspectiva. Rev Bras Reprod Anim Supl. 2009;6:98-103.

Benevides CMJ, Trigueiro IN, Santos MAF. Estudo da variação da produção e do teor de gordura, do leite de búfala (Raça Murrah) na microrregião de Catu-BA em 165 dias de lactação. Hig Aliment. 2001;15(80):100.
Bernardes 0. Bubalinocultura no Brasil: situação e importância econômica. Rev Bras Reprod Anim. 2007;31(3):293-8.

Borghese A (E). Buffalo production and research. Roma: Food and Agriculture Organization of the United Nations; 2005 [acesso 5 dez 2017]. Disponível em: https://tinyurl. com/y8qevq5l.

Broom DM. Animal welfare: an aspect of care, sustainability, and food quality required by the public. J Vet Med Educ. 2010;37(1):83-8.

Broom DM. Animal welfare: concepts, study methods and indicators. Rev Colom Cienc Pecua. 2011;24(3):306-21.

Broom DM. Indicators of poor welfare. $\mathrm{Br}$ Vet J. 1986;142(6):524-6.

Broom DM, Fraser AF. Comportamento e bem-estar de animais domésticos. Barueri: Manole; 2010.

Broom DM, Molento CFM. Bem-estar animal: conceito e questões relacionadas - revisão. Arch Vet Sci. 2004;9(2):1-11.

Carvalhal MVL, Sant'Anna AC, Páscoa AG, Jung J, Costa MJRP. The relationship between water buffalo cow temperament and milk yield and quality traits. Livest Sci. 2017;198:109-14.

Cavallina R, Roncoroni C, Campagna MC, Minero M, Canali E. Buffalo behavioural response to machine milking in early lactation. Ital J Anim Sci. 2008;7(3):287-95.

De Rosa G, Napolitano F, Grasso F, Pacelli C, Bordi A. On the development of a monitoring scheme of buffalo welfare at farm level. Ital J Anim Sci. 2005;4(2):115-25.

De Rosa G, Napolitano F, Grasso F, Bilancione A, Spadetta M, Pacelli $C$, et al. Welfare Quality $®$ : a pan-European integrated project including buffalo. Ital J Anim Sci. 2007a;6(Suppl 2):1360-3.

De Rosa G, Napolitano F, Saltalamacchia F, Bilancione A, Sabia E, Grasso F, et al. The effect of rearing system on behavioural and immune responses of buffalo heifers. Ital J Anim Sci. 2007b;6(Suppl 2):1260-3. 
Garcia PR. Sistema de avaliação do bem-estar animal para propriedades leiteiras com sistema de pastejo [dissertação]. Piracicaba: Universidade de São Paulo; 2013. 181 p.

Garcia AR, Matos LB, Lourenço Jr JB, Nahúm BS, Araújo $\mathrm{CV}$, Santos AX. Variáveis fisiológicas de búfalas leiteiras criadas sob sombreamento em sistemas silvipastoris. Pesq Agropec Bras. 2011;46(10):1409-14.

Grasso F, De Rosa G, Marsico I, Napolitano F, Migliori G, Bordi A. Welfare of buffalo heifers in relation to feeding and space allowance. Ital J Anim Sci. 2003;2(Suppl 1):148-50.

Grasso F, Terzano GM, De Rosa G, Tripaldi C, Napolitano F. Influence of housing conditions and calving distance on blood metabolites in water buffalo cows. Ital J Anim Sci. 2004;3(3):275-82.

IBGE (Instituto Brasileiro de Geografia e Estatística). PPM 2010: Rebanho bovino nacional cresce 2,1\% e chega a 209,5 milhões de cabeças. 2011 [acesso 14 jan 2018]. Disponível em: https://tinyurl.com/y994stvl.

IBGE (Instituto Brasileiro de Geografia e Estatística). Produção da Pecuária Municipal 2015. Prod Pec Munic. 2015;43:1-49. [acesso 18 maio 2018]. Disponível em: https://tinyurl.com/y89e5nfp.

Maton A, Daelemans J. Modern housing of cattle and their welfare. 11th International Congress on Agricultural Engineering; 4-8 set 1989; Dublin, Ireland. Rotterdam: Balkema; 1989, v.2. p. 921-5.

Mello RRC, Ferreira JE, Mello MRB. Aspectos da fisiologia da lactação em búfalas (Bubalus bubalis). Rev Bras Reprod Anim. 2016;40(1):8-16.

Mellor DJ. Operational Details of the Five Domains Model and Its Key Applications to the Assessment and Management of Animal Welfare. Animals (Basel). 2017;7(8):60.

Mellor DJ, Reid CSW. Concepts of animal well-being and predicting the impact of procedures on experimental animals. In: Baker RM, Jenkin G, Mellor DJ (EE.) Improving the Well-being of Animals in the Research Environment. Adelaide: ANZCCART; 1994. p. 3-18.
Napolitano F, De Rosa G, Grasso F, Pacelli C, Bordi A. Influence of space allowance on the welfare of weaned buffalo (Bubalus bubalis) calves. Livest Prod Sci. 2004;86(1-3):117-24.

Napolitano F, Pacelli C, Grasso F, Braghieri A, De Rosa G. The behaviour and welfare of buffaloes (Bubalus bubalis) in modern dairy enterprises. Animal. 2013;7(10):1704-13.

Nascimento C, Carvalho LOM. Criação de búfalos: alimentação, manejo, melhoramento e instalações. Brasília, DF: Embrapa-SPI; 1993. 403 p.

Polikarpus A, Napolitano F, Grasso F, Di Palo R, Zicarelli F, Arney D, et al. Effect of pre-partum habituation to milking routine on behaviour and lactation performance of buffalo heifers. Appl Anim Behav Sci. 2014;161:1-6.

Rodrigues CFC, Iapichini JECB, Liserre AM, Souza KB, Fachini C, Reichert RH. Oportunidades e desafios da bubalinocultura familiar da região sudoeste paulista. Tecnol Inov Agropecu (Tecnologia \& Inovação Agropecuária). 2008;100-9.

Sabia E, Napolitano F, De Rosa G, Terzano GM, Barile VL, Braghieri A, et al. Efficiency to reach age of puberty and behaviour of buffalo heifers (Bubalus bubalis) kept on pasture or in confinement. Animal. 2014;8(11):1907-16.

Saltalamacchia F, Tripaldi C, Castellano A, Napolitano F, Musto M, De Rosa G. Human and animal behaviour in dairy buffalo at milking. Anim Welf. 2007;16(2):139-42.

Teixeira LV, Bastianetto E, Oliveira DAA. Leite de búfala na indústria de produtos lácteos. Rev Bras Reprod Anim. 2005;29(2):96-100.

Thomas CS, Svennersten-Sjaunja K, Bhosrekar MR, Bruckmaier RM. Mammary cisternal size, cisternal milk and milk ejection in Murrah buffaloes. J Dairy Res. 2004;71(2):162-8.

Thomas CS, Bruckmaier RM, Ostensson K, SvennerstenSjaunja K. Effect of different milking routines on milkingrelated release of the hormones oxytocin, prolactin and cortisol, and on milk yield and milking performance in Murrah buffaloes. J Dairy Res. 2005;72(1):10-8. 
Tonhati H. Estudo da curva de lactação em bubalinos. 35a Reunião Anual Sociedade Brasileira de Zootecnia; jul 1998; Botucatu, SP. Botucatu: SBZ; 1998. p. 276-8.

Tripaldi C, De Rosa G, Grasso F, Terzano GM, Napolitano F. Housing system and welfare of buffalo (Bubalus bubalis) cows. Anim Sci. 2004;78(3):477-83.

Valle JLE. Características e usos do leite de bubalinos. $27^{\circ}$ Reunião Anual Sociedade Brasileira de Zootecnia; 22-27 jul 1990; Campinas, SP. Brasília: SBZ; 1990. p.739-43.
Vecchio D, D’angelo L, Luigi B, Galiero G, Romano F, Noschese R, et al. Bem-estar animal e biossegurança na produção de búfalos. IV Simpósio Paulista de Bubalinocultura; 3-5 maio 2018; Jaboticabal, SP. Jaboticabal: Unesp/FCAV; 2018. p. 20-5.

Welfare Quality ${ }^{\circledR}$. Assessment protocol for cattle. Lelystad: Welfare Quality ${ }^{\circledR}$ consortium; 2009. 142 p. 